\title{
A design of automatic boiler intelligent alert system based on the wireless AD hoc network
}

\author{
Shiping $\mathrm{Li}^{1},{ }^{\mathrm{a}}$ Maodong $\mathrm{Li}^{1},{ }^{\mathrm{b}}$ Wei Liu ${ }^{2},{ }^{\mathrm{c}}$ Wei Zhai ${ }^{1},{ }^{\mathrm{d}}$ Jinmei Lin ${ }^{1},{ }^{\mathrm{e}}$ Lian Wang ${ }^{1},{ }^{\mathrm{f}}$ \\ and Zhenling $\mathrm{Wu}^{1}{ }^{\mathrm{g}}$ \\ ${ }^{1}$ Guangzhou Special Pressure Equipment Inspection and Research Institute, Guangzhou 510100, \\ China; \\ ${ }^{2}$ Heilongjiang Province Electric Research Institue, Harbin 150030, China. \\ aboming21@163.com, ${ }^{\mathrm{b}}$ Imd92791@21cn.com, ${ }^{\mathrm{c}}$ 18249463685@163.com, ${ }^{\mathrm{d}}$ 234869961@qq.com \\ eJinmeiL2083@sina.com.cn, ${ }^{\mathrm{f}} 18410466 @ q q . c o m,{ }^{9}$ 623223878@qq.com
}

Keywords: automatic boiler, alert system, wireless, AD hoc network.

\begin{abstract}
A design of automatic boiler intelligent alert system based on the wireless AD hoc network is presented in this work for realizing unattended communication between the boiler and operators. This intelligent system was accessed to the original boiler alert system without reforming using the microprocessor module as monitoring node. The real-time alarm data can be stably transmitted to the operators with alarm terminal through ad Hoc Network and GPRS module. Actual tests show that this system owns good feasibility and effectiveness for ensuring the boiler security.
\end{abstract}

\section{Introduction}

Currently, the audio and video alarm system ${ }^{[1-2]}$ is widely applied by industrial boilers. However, the boiler operators cannot be alerted and respond timely when they are absent in the boiler room. According to the 《Safety Technical and Supervisary Specification of Steam Boiler》, class B automatic boiler can run in unattended operation with a regular inspection. In order to achieve unattended operation for the automatic boilers, an automatic boiler intelligent alert system ${ }^{[3]}$ based on the wireless $\mathrm{AD}$ hoc network was designed in the present work.

\section{System Configuration}

As showed in Fig. 1, this system mainly contains, routing node, alarm terminal and server terminal. Without remoulding the original control system of boiler, the monitoring node could be well accessed to the boiler alarm module to catch the alarm signal and sent alert information via wireless AD hoc network $^{[4]}$. Alarm data from monitoring nodes are took over, screened, handled and transmitted to the server terminal via GPRS. As a routing node can support a number of monitoring nodes, it will help reduce the cost of hardware and transmission. The alarm terminal then rapidly feeds back the alert information to boiler operator, greatly releasing the labor force as an effective unattended operation of the boiler can be realized. On the other hand, the server terminal collects and keeps the running data of boilers, providing data support for operation level evaluation of boiler equipment.

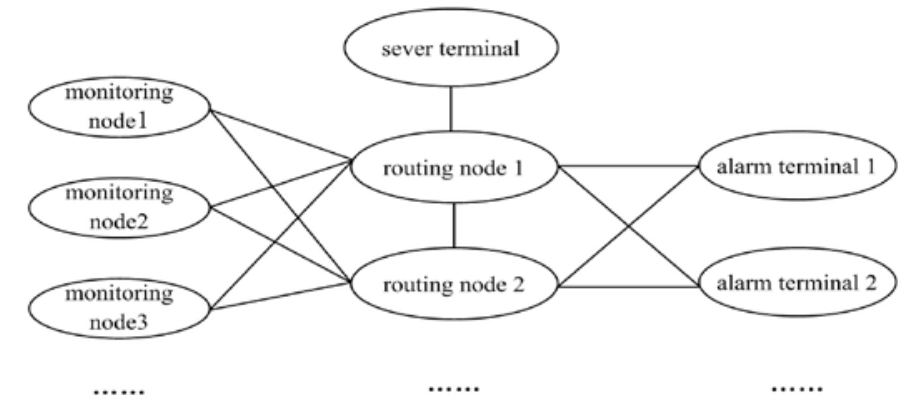

Fig. 1 Structure diagram of the alarm system 


\section{System Design}

Monitoring Node. The monitoring node mainly consists of microprocessor, signal acquisition module, power module and wireless communication module. The existing alarm module generally works via audio and video alarm ${ }^{[5]}$, that is, reminds the operator using flashing lights and ring tones. In parallel with the alarm system, the signal acquisition module converts the electrical level of the alarm system into electrical signal which can be recognized by microprocessor. Then, the microprocessor broadcasts the running state of a boiler through wireless module intermittently.

Routing node. Routing node includes microprocessors, wireless module and network module. Routing node mainly aims to transit radio signals and extend the broadcast radius of wireless signal. Simultaneously, the alert signal of boilers is sent and saved in the sever terminal through network communication including Wi-fi and GPRS.

Alarm terminal. Alarm terminal composes of low power consumption microprocessor, display module, warning module, power module and the wireless communication module. Microprocessor is wake up intermittently and then receives data from routing or monitoring node. The warning module will produce a warning in the form of vibration and sound if the signal is abnormal ${ }^{[6]}$.

Server terminal. The administrator can momentarily log in the automatic alarm monitoring system to check and analysis the alarm data of the boilers. The server terminal mainly works to receive the request from routing nodes around the network. On the other hand, it receives the query request of the page platform and presents the summarized data to the administrator.

\section{Node Agreement}

System communication agreement. The communication protocol as shown in Table 1was applied in order to standardize the communication between nodes. The communication routes between nodes are divided into wireless and network. The current information is to be broadcasted by the monitoring and routing nodes every 5 ms through wireless module. Ether the boiler status change or the time interval is beyond $30 \mathrm{~min}$, the monitoring and routing nodes will communicate the server terminal through a communication network to check the running condition of the equipment.

Table 1 Communication protocol

\begin{tabular}{lcc}
\hline \multicolumn{1}{c}{ Frame } & Data length/Byte & State \\
\hline Start flag & 2 & Start flag of data frames \\
Frame category & 1 & Data collection or configuration order \\
Node ID & 4 & 32 bite number \\
Frame number & 1 & Differentiating repetitive frame \\
Boiler operation information & 5 & Definition of boiler alarm information \\
Frame check & 1 & Information validity checking \\
Ending flag & 2 & Ending flag of data frames \\
\hline
\end{tabular}

\section{Working ways of the monitoring and routing nodes}

Monitoring nodes have two working modes containing setting mode and running mode, which can be switched. The users can set ID and communication frequency of the monitoring nodes ${ }^{[7]}$ in the setting mode. When in running mode, the monitoring nodes will send the operation status of boilers through wireless module in real time.

In order to extend the radius of broadcast, routing nodes will sent the received wireless data which is checked without any errors. Simultaneously, the routing nodes will contact with the sever terminal intermittently according to the communication protocol, to remain the system stability with consuming low network traffic. 


\section{Working way of the alarm terminal}

The alarm terminal wakes up and receives wireless signals every 30s. To reducing the energy consumption, the alarm terminal goes to sleep when receives a normal running signal of boiler. While receiving the alarm signal, the alarm terminal reminds the boiler operator in the firm of vibration and sound.

\section{Telecommunication Test Based on Wireless Module}

The wireless module, contributing to data transmission between wireless modules, must have timeliness and reliability. In order to ensure the battery life, the wireless module should have a characteristic of low power consuming. Due to the long-time operation and huge volume of data, a low cost for data transmission is vital to reduce the equipment operation cost. On the other hand, the wireless module must own strong power for barrier penetration in order to expand the activity scope of operators carrying the alarm module ${ }^{[8]}$. In a word, the wireless module must have properties of low energy consumption, low cost, strong penetration power and so on.

Currently, some mature module technilogise ${ }^{[9]}$ includes Zigbee, bluetooth low energy (BLE) and $433 \mathrm{M}$ transceiver module technology. The properties of each technology are shown in Table 2.

Table 2 Wireless transmission technologies

\begin{tabular}{lcccc}
\hline Technology & $\begin{array}{c}\text { Frequency } \\
\text { band }\end{array}$ & $\begin{array}{c}\text { Transmission d } \\
\text { istance }\end{array}$ & Transmission rate & Characteristic \\
\hline Zigbee & $2.4 \mathrm{G}$ & $1 \sim 2 \mathrm{~km}$ & $250 \mathrm{kbps}$ & $\begin{array}{c}\text { close, low-complexity, low power } \\
\text {, low data rate, low cost } \\
\text { low cost, close, low power, } \\
\text { interoperability }\end{array}$ \\
BLE & $2.4 \mathrm{G}$ & $100 \mathrm{~m}$ & $48 \mathrm{kbps}$ & $\begin{array}{c}\text { low power, strong receptivity, } \\
\text { strong penetrability }\end{array}$ \\
$433 \mathrm{MHz}$ & $433 \mathrm{MHz}$ & $1 \sim 2 \mathrm{~km}$ & $100 \mathrm{kbps}$ & \\
\hline
\end{tabular}

\section{Telecommunication testing}

The wireless module communication test was conducted in an area of $1500 \mathrm{~m} \times 1500 \mathrm{~m}$ in an industrial park. As shown in figure 2, there are three big conjoined building ABC, a small building D, an empty playground $\mathrm{E}$ and a parking lot $\mathrm{F}$. Monitoring and routing nodes using three transmission technologies are set up on the first floor in building A and C.

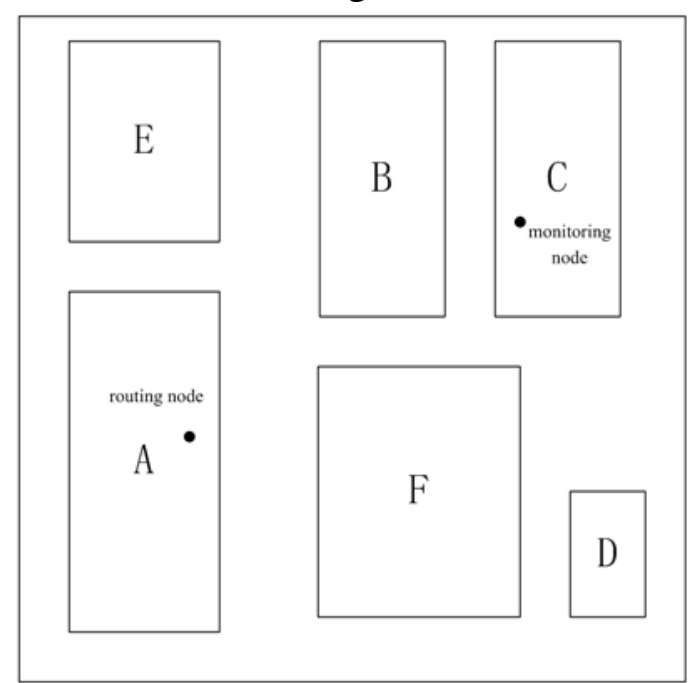

Fig. 2 Schematic diagram of the test field

During the test process, signal strength and signal loss of alarm terminal using different transmission technologies was tested in various position of this park. Telecommunicating performance of three transmission technologies are shown in Figure 3. 


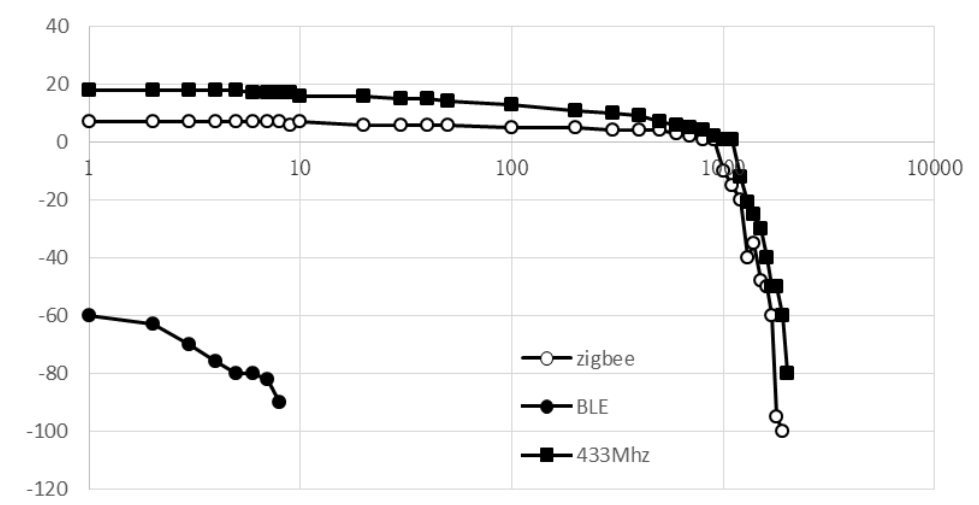

Fig. 3 Influence of communication distance on signal

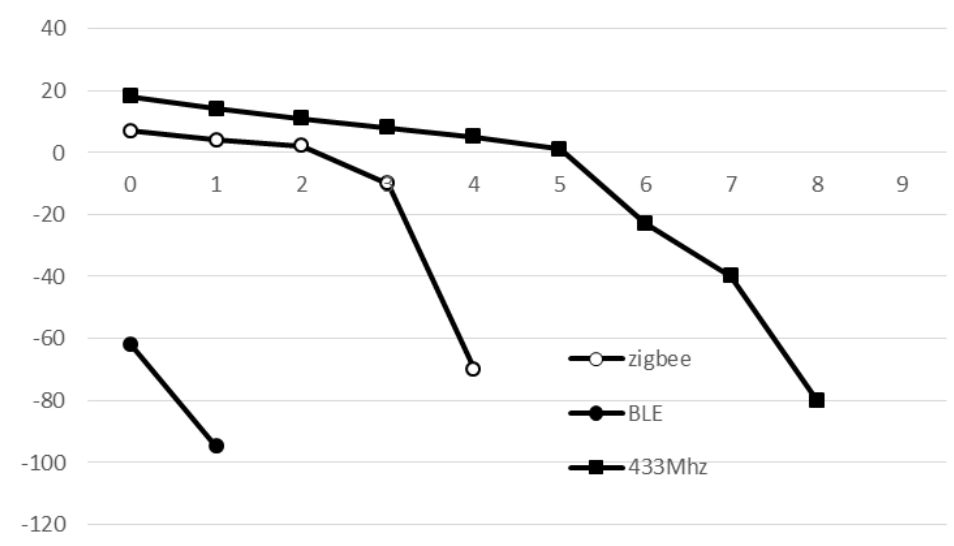

Fig. 4 Influence of deaden walls on signal

The telecommunication test shows that the wireless module using $2.4 \mathrm{G}$ bands of Zigbee and Bluetooth with low energy consumption (BLE) technology has strong advantage in transmission rate. However, it owns weak ability of bypassing obstacles. There exists strong signal attenuation due to the obstacles such as walls between transmitting terminal and receiving terminal, causing that signal cannot achieve the nominal transmission distance.

The wireless module with $433 \mathrm{MHz}$ wireless technology displays stable communication performance, mainly due to its long wavelength. A stable communication can be still remained and the communication rate can meet the requirement for detecting the alarm system even the signal is cut off by obstacles like walls.

\section{Conclusion}

This work studied a design of automatic boiler intelligent alert system which consists of monitoring nodes, routing nodes, alarm terminal and server terminal integrated with the wireless AD hoc networks and GPRS, making unattended communication a reality between the automatic boiler and operators. Field test and results analysis based on the wireless communication modules show that wireless module with $433 \mathrm{MHz}$ wireless technology has advantages of long transmission distance and strong ability for bypassing obstacles. To further promote its advantages like low power consumption, low cost, strong penetrating, an appropriate dormant agreement to effectively prolong the battery life can be taken into consideration.

\section{References}

[1] Weihui DUAN. Computer automatic control system in boiler [J]. Automation \& Instrumentation, 2010(2). 
[2] Yinjuan Chen. Design of M onitoring Management System for Operation Conditions of Boiler [D]. Lanzhou Jiaotong University, 2014.

[3] Shuqing Ma, Qinghui Fang, Dongpeng Wang. Auto-alarming system of liquid level and temperature in boiler [J]. Journal of Transducer technology, 2000, 19(5).

[4] Yue-xin Li, Ming Zhu. Study on capacity of route aggregate networks based on slotted transmission protocol [J]. Computer Science, 2015, 42(8).

[5] Zaijun Su, Lihong Ma, Li Li. Research on safety and alarm system for industrial boilers and kilns Power System Engineering [J]. Computer Science, 2011, 17(4).

[6] Xiyun Huang, Mingxue Li. Design of detecting and warning system of boiler heat supply temperature [J]. Journal of Heilongjiang Institute of Science \& Technology, 2004, 14(6).

[7] Qiangsheng Dai, Shaorong Wang. Application of SimpliciTI Based Wireless Ad-hoc Network in Intelligent Monitoring and Control System for Distribution Networks [J]. Automation of Electric Power Systems, 2014(7).

[8] Lei Zhang, Xuehui Wang, Wenhua Dou. A Broadcast Algorithm based on Primary-Secondary Dominators for Wireless Ad Hoc Networks and Its Optimization [J]. Chinese Journal of Computers, 2006,29(11).

[9] Shenqiu Liu, Yigong Li, Cuixia Yang. The anti-interference ability of micro-power wireless ad-hoc network [C] 2013. 